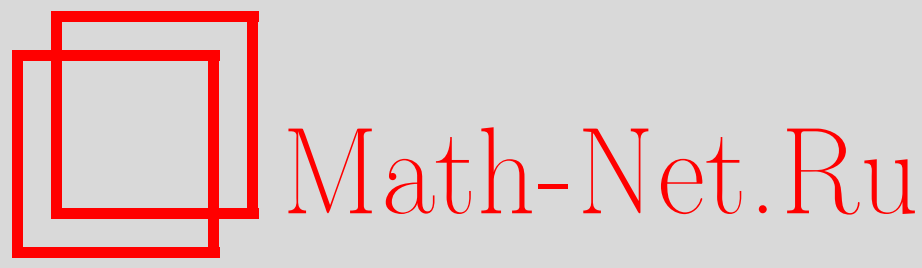

Т. Такаги, Новая обобщенная теорема Вика в конформной теории поля, ТМФ, 2017, том 192, номер 2, 335-347

DOI: https://doi.org/10.4213/tmf9297

Использование Общероссийского математического портала Math-Net.Ru подразумевает, что вы прочитали и согласны с пользовательским соглашением http: //www.mathnet.ru/rus/agreement

Параметры загрузки :

IP : 54.224 .60 .19

26 апреля 2023 г., 15:19:43

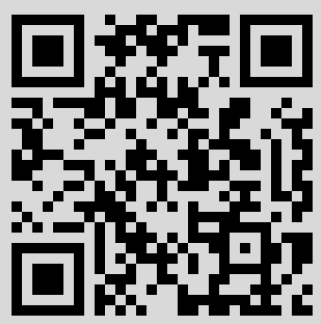


Том 192, № 2

август, 2017

(C) 2017 г. Т. Такаги*

\title{
НОВАЯ ОБОБЩЕННАЯ ТЕОРЕМА ВИКА В КОНФОРМНОЙ ТЕОРИИ ПОЛЯ
}

\begin{abstract}
Излагается новая обобщенная теорема Вика для взаимодействующих полей в двумерной конформной теории поля. Кратко обсуждается ее связь с тождеством Борхердса и ее вывод аналитическим методом. Также представлены вычисления операторных разложений путем использования обобщенной теоремы Вика, содержащей фермионные поля.
\end{abstract}

Ключевые слова: операторное разложение, вертексная алгебра, тождество Борчердса, фермионные поля.

DOI: https://doi.org/10.4213/tmf9297

\section{1. ВВЕДЕНИЕ}

Хорошо известная обобщенная теорема Вика для взаимодействующих полей в двумерной конформной теории поля (КТП) была впервые сформулирована в 1988 г. в работе [1], более точное ее описание можно найти в стандартном учебнике по КТП [2]. Эта теорема формулируется следующим образом:

$$
\overparen{A(z)(B C})(w)=\frac{1}{2 \pi \sqrt{-1}} \oint_{C_{w}} \frac{d x}{x-w}\{\overleftrightarrow{A(z) B}(x) C(w)+B(x) \overleftrightarrow{A(z) C}(w)\} .
$$

Здесь $A, B$ и т. д. суть операторы киральных конформных полей,

$$
(B C)(w)=: B(w) C(w):
$$

означает нормально упорядоченное произведение, $\overleftrightarrow{A(z) B}(x)$ - спаривание, т. е. сингулярная часть операторного разложения и $C_{w}$ - окружающий точку $w$ контур исчезающе малого радиуса.

Данная работа была поддержана Japan Society for the Promotion of Science (грант KAKENHI JP25400122).

*Department of Applied Physics, National Defense Academy, Kanagawa, Japan. E-mail: takagi@nda.ac.jp 
Возникает естественный вопрос, существует ли аналогичное выражение для спаривания $(A B)(z) C(w)$, в котором нормально упорядоченное произведение операторов стоит слева, а взятый отдельно оператор - справа. Насколько известно автору, пока никто не дал корректного ответа на этот вопрос. Мы попытались найти этот ответ и получили следующее выражение [3]:

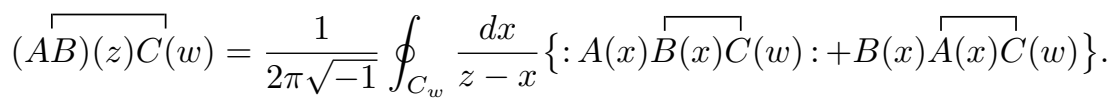

Здесь нормальное упорядочение в первом слагаемом под интегралом нужно для того, чтобы отбросить сингулярные члены, которые возникают в операторном разложении оператора $A(x)$ с коэффициентами спаривания, стоящими справа от него. Заметим, что даже в (1) можно заменить второе слагаемое на : $B(x) \overparen{A(z) C}(w):$, отчего две формулы становятся более очевидно схожими, но этого обычно не делают, потому что сингулярные члены автоматически отбрасываются в результате интегрирования в (1). Весьма примечательно, что эта действительно простая формула до сих пор не найдена, хотя прошло уже более чем 25 лет после формулировки теоремы (1).

В разделе 2 мы кратко описываем доказательство обощенной теоремы Вика (2), которое основано на тождестве Борчердса. Также мы приводим набросок ее доказательства аналитическим методом. В разделе 3 мы приводим примеры вычисления операторных разложений путем использования обощенной теоремы Вика, содержащей фермионные поля. В целях упрощения изложения почти все строгие математические рассуждения опущены, интересующийся читатель может найти их в работе [3].

\section{2. ТЕОРЕМА ВИКА КАК ОСОБЫЙ СЛУЧАЙ ТОЖДЕСТВА БОРЧЕРДСА}

2.1. Обозначения. Чтобы математически описать двумерные киральные квантовые поля, воспользуемся формулировкой Мацуо и Нагамото [4]. Запишем оператор $A(y)$ как формальный степенной ряд и выделим в нем положительную и отрицательную части:

$$
A(y)=\sum_{n \in \mathbb{Z}} A_{n} y^{-n-1}=\left(\sum_{n \geqslant 0}+\sum_{n<0}\right) A_{n} y^{-n-1}=A(y)_{+}+A(y)_{-} .
$$

Здесь коэффициенты $A_{n}$ представляют собой линейные преобразования в некотором векторном пространстве $M$. Нормально упорядоченное произведение операторов $A(y)$ и $B(z)$ определяется как

$$
: A(y) B(z):=A(y)_{-} B(z)+B(z) A(y)_{+} \cdot
$$

Далее окажется, что оно регулярно при $y=z$ в некотором смысле.

Теперь мы хотим сделать важное замечание, касающееся так называемого $n$-го произведения. Оно задается следующей формулой для неотрицательного $n$ :

$$
A(z)_{(n)} B(z)=\operatorname{Res}_{y=0}[A(y), B(z)](y-z)^{n} .
$$


При этом спаривание может быть определено как

$$
\stackrel{\vec{A}(y) B}{B}(z)=\sum_{n=0}^{\infty} \frac{A(z)_{(n)} B(z)}{(y-z)^{n+1}} .
$$

Мы имеем следующее операторное разложение в области $|y|>|z|$ :

$$
A(y) B(z)=\overparen{A(y) B}(z)+: A(y) B(z): .
$$

Слегка модифицируя выражение (5), мы можем также задать $n$-е произведения для отрицательных $n$, в частности мы нередко будем пользоваться тем, что (-1)-е произведение равно нормально упорядоченному произведению:

$$
A(z)_{(-1)} B(z)=: A(z) B(z):
$$

Заметим, что мы часто будем использовать определение (5) даже для отрицательных $n$, понимая его просто как сокращенное обозначение.

2.2. Тождество Борчердса. Запишем сначала обычное тождество Якоби:

$$
[[A, B], C]=[A,[B, C]]-[B,[A, C]] .
$$

Из него Мацуо и Нагамото вывели тождество

$$
\begin{aligned}
& \sum_{i=0}^{\infty}\left(\begin{array}{l}
p \\
i
\end{array}\right)\left(A_{(r+i)} B\right)_{(p+q-i)} C= \\
& \quad=\sum_{i=0}^{\infty}(-1)^{i}\left(\begin{array}{c}
r \\
i
\end{array}\right)\left(A_{(p+r-i)}\left(B_{(q+i)} C\right)-(-1)^{r} B_{(q+r-i)}\left(A_{(p+i)} C\right)\right) .
\end{aligned}
$$

Здесь мы опустили аргументы операторов, которые в дальнейшем полагаем равными $w$. Маленькие скобки в нижних индексах означают, что мы берем произведение дважды. Это так называемое тождество Борчердса, известное как одна из аксиом вертексной алгебры [5]. Мацуо и Нагамото доказали, что оно верно для всех целых $p, q, r$, если сделать предположение о том, что квантовые поля обладают неким свойством, названным локальностью.

2.3. Некоммутативная формула Вика, полученная Кацем. Рассмотрим частный случай тождества при $r=0$ и $q=-1$ для неотрицательных $p$ :

$$
A_{(p)}\left(B_{(-1)} C\right)=\left(A_{(p)} B\right)_{(-1)} C+B_{(-1)}\left(A_{(p)} C\right)+\sum_{i=0}^{p-1}\left(\begin{array}{c}
p \\
i
\end{array}\right)\left(A_{(i)} B\right)_{(p-i-1)} C .
$$

В своей знаменитой книге [6] Кац получил это тождество своим методом и назвал его "некоммутативная формула Вика". Как указал Кац, для свободных полей последнее слагаемое исчезает и формула сводится к обычной теореме Вика для свободных полей. Также он отметил ее эквивалентность обобщенной формуле Вика (1). Доказательство этой эквивалентности можно найти в работе [3]. 


\section{4. Алгебраическое доказательство новой обобщенной теоремы Вика.} Покажем, что наша новая обобщенная теорема Вика (2) эквивалентна еще одному частному случаю тождества Борчердса. Начнем с некоторых формул. Нетрудно доказать, что для любого целого $n$

$$
\partial A(w)_{(n)} B(w)=-n A(w)_{(n-1)} B(w) .
$$

Заменяя $n$ на $-n$ и используя данное соотношение, мы получаем

$$
n ! A_{(-n-1)} B=(n-1) ! \partial A_{(-n)} B=(n-2) ! \partial^{2} A_{(-n+1)} B=\cdots=\partial^{n} A_{(-1)} B,
$$

следовательно, для любого неотрицательного целого $n$

$$
: \partial^{(n)} A B:=A_{(-n-1)} B .
$$

Здесь $\partial^{(n)}=\partial^{n} / n !$. Теперь первое слагаемое : $A(x) \stackrel{B(x) C}{ }(w):$ под интегралом в нашей формуле (2) может быть записано как

$$
: A(x) B(x) C(w):=\sum_{i=0}^{\infty} \frac{: A(x)\left(B(w)_{(i)} C(w)\right):}{(x-w)^{i+1}}=\sum_{i=0}^{\infty} \sum_{j=0}^{\infty} \frac{A_{(-j-1)}\left(B_{(i)} C\right)}{(x-w)^{i-j+1}} .
$$

Здесь мы заменили оператор $A(x)$ его разложением Тейлора в окрестности точки $w$ и затем использовали формулу (11). После умножения на $(z-x)^{-1}$ и интегрирования в окрестности точки $w$ по $x$ выживают только сингулярные члены. Затем мы заменяем все $x$ на $z$. Положив $i=q+j$, в итоге получаем

$$
\begin{aligned}
\frac{1}{2 \pi \sqrt{-1}} \oint_{C_{w}} \frac{d x}{z-x}\{: A(x) \overparen{B(x) C}(w):\} & =\sum_{i=0}^{\infty} \sum_{j=0}^{i} \frac{A_{(-j-1)}\left(B_{(i)} C\right)}{(z-w)^{i-j+1}}= \\
& =\sum_{q=0}^{\infty} \sum_{j=0}^{\infty} \frac{A_{(-j-1)}\left(B_{(q+j)} C\right)}{(z-w)^{q+1}} .
\end{aligned}
$$

Рассмотрим второе слагаемое в подынтегральном выражении в нашей формуле (2). Выделим в нем сингулярную и регулярную части:

$$
B(x) \overparen{A(x) C}(w)=\underset{\underbrace{}}{B(x)}(\overparen{A(x)} C(w))+: B(x) \overparen{A(x) C}(w): .
$$

В силу тех же аргументов, что и выше, после взятия интеграла мы получаем для этого слагаемого выражение

$$
\sum_{i=0}^{\infty} \sum_{j=0}^{\infty} \frac{B_{(j)}\left(A_{(i)} C\right)}{(z-w)^{i+j+2}}+\sum_{i=0}^{\infty} \sum_{j=0}^{i} \frac{B_{(-j-1)}\left(A_{(i)} C\right)}{(z-w)^{i-j+1}}
$$

Мы можем объединить эти две суммы в одну, заменяя индекс суммирования $j$ в первой сумме на $-j-1$. Положив затем $j=q-i-1$, мы получаем

$$
\begin{aligned}
\frac{1}{2 \pi \sqrt{-1}} \oint_{C_{w}} \frac{d x}{z-x}\{B(x) \overparen{A(x) C}(w)\} & =\sum_{i=0}^{\infty} \sum_{j=-i-1}^{\infty} \frac{B_{(j)}\left(A_{(i)} C\right)}{(z-w)^{i+j+2}}= \\
& =\sum_{q=0}^{\infty} \sum_{i=0}^{\infty} \frac{B_{(q-i-1)}\left(A_{(i)} C\right)}{(z-w)^{q+1}} .
\end{aligned}
$$


Из полученных результатов мы находим, что наша новая обобщенная теорема Вика (2) эквивалентна следующему утверждению:

$$
\left(A_{(-1)} B\right)_{(q)} C=\sum_{i=0}^{\infty}\left(A_{(-i-1)}\left(B_{(q+i)} C\right)+B_{(q-i-1)}\left(A_{(i)} C\right)\right) .
$$

Как можно видеть, это соотношение есть не что иное, как частный случай тождества Борчердса при $p=0, r=-1$.

\section{5. Аналитическое доказательство новой обобщенной теоремы Вика.} Как показано выше, получив выражение для нашей новой обощенной теоремы Вика, можно проверить ее справедливость путем простого вычисления интегралов и последующего сравнения с тождеством Борчердса. Однако возникает естественный вопрос: каким образом можно прийти к данному выражению? В оставшейся части данного раздела мы намерены дать ответ на этот вопрос. Мы приводим набросок доказательства нашей новой формулы с помощью аналитического метода.

По определению вложенное произведение $\left(A_{(-1)} B\right)_{(p)} C$ задается как дважды взятый вычет вложенного коммутатора. Перепишем это выражение, используя контурный интеграл:

$$
\begin{aligned}
& \left.\left(A_{(-1)} B\right)_{(p)} C\right)=\operatorname{Res}_{x=0} \operatorname{Res}_{y=0}[[A(y), B(x)], C(w)](y-x)^{-1}(x-w)^{p}= \\
& =\frac{1}{(2 \pi \sqrt{-1})^{2}}\left\{\oint_{C_{3}} d x \oint_{C_{4}} d y-\oint_{C_{4}} d x \oint_{C_{3}} d y-\oint_{C_{1}} d x \oint_{C_{2}} d y+\oint_{C_{2}} d x \oint_{C_{1}} d y\right\} A(y) B(x) C(w) \times \\
& \times \frac{(x-w)^{p}}{y-x} .
\end{aligned}
$$

Здесь четыре слагаемых возникают из разложения вложенного коммутатора. Контуры $C_{1,2,3,4}$ суть окружности с центром в начале координат и возрастающими радиусами, причем точка $w$ лежит между $C_{2}$ и $C_{3}$.

Пусть контуры $C_{3}$ и $C_{4}$ стягиваются к $C_{1}$ и $C_{2}$ соответственно, так что они взаимно уничтожают друг друга. Но, конечно, при такой деформации они захватывают сингулярность в точке $w$. В результате мы имеем следующее выражение:

$$
\left.\left(A_{(-1)} B\right)_{(p)} C\right)=\frac{1}{(2 \pi \sqrt{-1})^{2}}\left\{\oint_{C_{w}^{>}} d y \oint_{C_{w}^{<}} d x-\oint_{C_{w}^{>}} d x \oint_{C_{w}^{<}} d y\right\} A(y) B(x) C(w) \frac{(x-w)^{p}}{y-x}
$$

контуры $C_{w}^{>}$and $C_{w}^{<}$показаны на рис 1 . Умножая на $(z-w)^{-p-1}$ и суммируя по $p$ от нуля до бесконечности, мы получаем

$$
\begin{aligned}
(A B)(z) C(w)=\frac{1}{(2 \pi \sqrt{-1})^{2}}\left\{\oint_{C_{w}^{>}} d y \oint_{C_{w}^{<}} d x-\oint_{C_{w}^{>}} d x \oint_{C_{w}^{<}} d y\right\} A(y) B(x) C(w) \times \\
\times \frac{1}{(y-x)(z-x)} .
\end{aligned}
$$

Рассмотрим сначала второе слагаемое. С учетом общего знака минус внутренний интеграл задается выражением

$$
\frac{1}{2 \pi \sqrt{-1}} \oint_{C_{w}^{<}} \frac{d y}{x-y} A(y) B(x) C(w)=B(x) \stackrel{A(x) C}{C}(w) .
$$




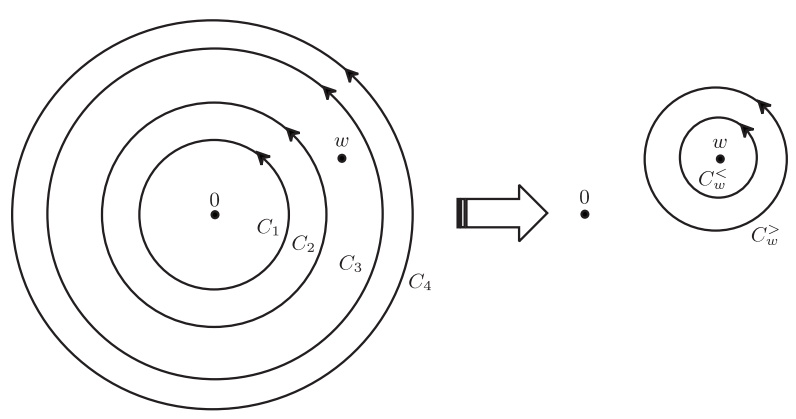

Рис. 1. Деформация контуров.

Так как этот интеграл берется по переменной $y$ вокруг точки $w$, он захватывает сингулярную часть операторного разложения произведения $A(y) C(w)$ и затем заменяет $y$ на $x$. В итоге получается результат, приведенный выше. После взятия внешнего интеграла по $x$ мы получаем второе слагаемое в нашей обобщенной теореме Вика.

Теперь рассмотрим первое слагаемое. Запишем множитель $(y-x)^{-1}(z-x)^{-1}$ в виде разности:

$$
\frac{1}{(y-x)(z-x)}=\frac{1}{z-y}\left(\frac{1}{y-x}-\frac{1}{z-x}\right) .
$$

Тогда внутренний интеграл по $x$ принимает вид

$$
\frac{1}{2 \pi \sqrt{-1}}\left(\oint_{C_{w}^{<}} \frac{d x}{y-x}-\oint_{C_{w}^{<}} \frac{d x}{z-x}\right) A(y) B(x) C(w)=A(y) \overrightarrow{B(y) C}(w)-A(y) B(z) C(w) .
$$

В точности так же, как при предыдущих расчетах, получаем результат интегрирования, аналогичный выписанному выше. Рассмотрим внешний интеграл по $y$ :

$$
\begin{gathered}
\frac{1}{2 \pi \sqrt{-1}} \oint_{C_{w}} \frac{d y}{z-y}(A(y) \overrightarrow{B(y) C}(w)-A(y) \overrightarrow{B(z) C}(w))= \\
=\frac{1}{2 \pi \sqrt{-1}} \oint_{C_{w}} \frac{d y}{z-y}: A(y) B(y) C(w): .
\end{gathered}
$$

Регулярная часть второго слагаемого исчезает, поскольку она не имеет сингулярности при $y=w$. Сингулярные части первого и второго слагаемых после интегрирования взаимно сокращаются. Таким образом, остается только регулярная часть первого слагаемого, как и выше. Заменяя $y$ на $x$, мы получаем первое слагаемое в нашей обобщенной теореме Вика (2).

2.6. Пример 1: свободные бозоны. Пусть $J(z)$ - ток свободных бозонных полей, удовлетворяющий уравнению

$$
J(z) J(w)=\frac{1}{(z-w)^{2}}+: J(z) J(w): .
$$

Определим тензор энергии-импульса как

$$
T(z)=\frac{1}{2}: J(z) J(z):=\frac{1}{2}(J J)(z) .
$$


Тогда, используя тождество (1), мы имеем

$$
\begin{aligned}
\overrightarrow{J(z) T}(w) & =\frac{1}{2} J(z)(J J)(w)= \\
& =\frac{1}{2} \cdot \frac{1}{2 \pi \sqrt{-1}} \oint_{C_{w}} \frac{d x}{x-w}\left\{\frac{1}{(z-x)^{2}} J(w)+J(x) \frac{1}{(z-w)^{2}}\right\}=\frac{J(w)}{(z-w)^{2}} .
\end{aligned}
$$

С учетом тождества (2) получаем

$$
\begin{aligned}
\overparen{T(z) T}(w) & =\frac{1}{2}(J J)(z) T(w)= \\
& =\frac{1}{2} \cdot \frac{1}{2 \pi \sqrt{-1}} \oint_{C_{w}} \frac{d x}{z-x}\left\{: J(x) \frac{J(w)}{(x-w)^{2}}:+J(x) \frac{J(w)}{(x-w)^{2}}\right\}= \\
& =\frac{1}{2} \cdot \frac{1}{2 \pi \sqrt{-1}} \oint_{C_{w}} \frac{d x}{z-x}\left\{\frac{1}{(x-w)^{4}}+\frac{2: J(x) J(w):}{(x-w)^{2}}\right\}= \\
& =\frac{1}{2}\left\{\frac{1}{(z-w)^{4}}+\frac{2(J J)(w)}{(z-w)^{2}}+\frac{2: \partial J(w) J(w):}{z-w}\right\}= \\
& =\frac{1 / 2}{(z-w)^{4}}+\frac{2 T(w)}{(z-w)^{2}}+\frac{\partial T(w)}{z-w} .
\end{aligned}
$$

Это операторное разложение КТП с центральным зарядом $c=1$. В ходе преобразований мы использовали соотношение

$$
: \partial J(w) J(w):=: J(w) \partial J(w):
$$

справедливое в силу следующей формулы кососимметричности [4]:

$$
B(z)_{(m)} A(z)=\sum_{i=0}^{\infty}(-1)^{m+i+1} \partial^{(i)}\left(A(z)_{(m+i)} B(z)\right) .
$$

\section{3. ПРИМЕНЕНИЯ К ОПЕРАТОРНОМУ РАЗЛОЖЕНИЮ ДЛЯ ФЕРМИОННЫХ ПОЛЕЙ}

3.1. Расширение обощенной теоремы Вика на случай присутствия фермионных полей. Пусть $p(A)$ - четность произвольного поля $A$, т. е. $p(A)=0$ $(p(A)=1)$, если $A$ - бозонное (соответственно фермионное) поле [6]. При включении фермионных полей обощенную теорему Вика следует расширить до

$$
\begin{aligned}
& \overparen{A(z)(B C)}(w)=\frac{1}{2 \pi \sqrt{-1}} \oint_{C_{w}} \frac{d x}{x-w}\left\{\overleftrightarrow{\left.A(z) B(x) C(w)+(-1)^{p(A) p(B)} B(x) \overrightarrow{A(z) C}(w)\right\}}\right. \\
& \left(\overrightarrow{A B)(z) C}(w)=\frac{1}{2 \pi \sqrt{-1}} \oint_{C_{w}} \frac{d x}{z-x}\left\{: A(x) B(x) C(w):+(-1)^{p(A) p(B)} B(x) \overrightarrow{A(x) C}(w)\right\} .\right.
\end{aligned}
$$

Далее мы приведем два наиболее простых, но нетривиальных примера, чтобы проиллюстрировать справедливость этих соотношений. 
3.2. Пример 2: свободные фермионы. Пусть $\psi(z)$ - поле свободных фермионов, удовлетворяющее уравнению

$$
\psi(z) \psi(w)=\frac{1}{z-w}+: \psi(z) \psi(w): .
$$

Определим тензор энергии-импульса $T(z)$ как

$$
T(z)=-\frac{1}{2}: \psi(z) \partial \psi(z):
$$

С помощью соотношения (19) мы получаем

$$
\begin{aligned}
\overrightarrow{\psi(z) T}(w) & =-\frac{1}{2} \frac{1}{2 \pi \sqrt{-1}} \oint_{C_{w}} \frac{d x}{x-w}\{\overrightarrow{\psi(z) \psi}(x) \partial \psi(w)-\psi(x) \overrightarrow{\psi(z) \partial \psi}(w)\}= \\
& =-\frac{1}{2} \frac{1}{2 \pi \sqrt{-1}} \oint_{C_{w}} \frac{d x}{x-w}\left\{\frac{\partial \psi(w)}{z-x}-\frac{\psi(x)}{(z-w)^{2}}\right\}= \\
& =\frac{\psi(w) / 2}{(z-w)^{2}}-\frac{\partial \psi(w) / 2}{z-w}
\end{aligned}
$$

следовательно,

$$
\partial \overleftrightarrow{\psi(z) T}(w)=-\frac{\psi(w)}{(z-w)^{3}}+\frac{\partial \psi(w) / 2}{(z-w)^{2}} .
$$

Далее с помощью соотношения (20) мы получаем

$$
\overparen{T(z) T}(w)=-\frac{1}{2} \frac{1}{2 \pi \sqrt{-1}} \oint_{C_{w}} \frac{d x}{z-x}\{: \psi(x) \partial \overleftrightarrow{\psi(x) T}(w):-\partial \psi(x) \overrightarrow{\psi(x) T}(w)\} .
$$

Два слагаемых в подынтегральном выражении можно записать как

$$
\begin{aligned}
& : \psi(x) \partial \overrightarrow{\psi(x) T}(w):=\frac{: \psi(w) \psi(x):}{(x-w)^{3}}+\frac{1}{2} \frac{: \psi(x) \partial \psi(w):}{(x-w)^{2}}, \\
& -\partial \psi(x) \overparen{\psi(x) T}(w)=\frac{-1 / 2}{(x-w)^{2}} \partial \psi(x) \psi(w)+\frac{1 / 2}{x-w} \partial \psi(x) \partial \psi(w)= \\
& =\frac{-1 / 2}{(x-w)^{4}}+\frac{1}{2} \frac{: \psi(w) \partial \psi(x):}{(x-w)^{2}}+\frac{1}{2} \frac{\partial \psi(x) \partial \psi(w):}{x-w} .
\end{aligned}
$$

Суммируя их и разлагая числители в окрестности точки $x-w$, мы получаем

$$
\begin{aligned}
& : \psi(x) \partial \overrightarrow{\psi(x) T}(w):-\partial \psi(x) \overrightarrow{\psi(x) T}(w)= \\
& \quad=\frac{-1 / 2}{(x-w)^{4}}+\frac{2: \psi(w) \partial \psi(w):}{(x-w)^{2}}+\frac{: \psi(w) \partial^{2} \psi(w):}{x-w}+\cdots
\end{aligned}
$$

где многоточием обозначены члены, регулярные при $x=w$. Следовательно,

$$
\begin{aligned}
\overparen{T(z) T}(w) & =-\frac{1}{2}\left\{\frac{-1 / 2}{(z-w)^{4}}+\frac{2: \psi(w) \partial \psi(w):}{(z-w)^{2}}+\frac{: \psi(w) \partial^{2} \psi(w):}{z-w}\right\}= \\
& =\frac{1 / 4}{(z-w)^{4}}+\frac{2 T(w)}{(z-w)^{2}}+\frac{\partial T(w)}{z-w} .
\end{aligned}
$$

Это операторное разложение КТП с центральным зарядом $c=1 / 2$. 
Можно также использовать соотношение (20), чтобы вычислить

$$
\begin{aligned}
\overparen{T(z) \psi}(w) & =-\frac{1}{2} \frac{1}{2 \pi \sqrt{-1}} \oint_{C_{w}} \frac{d x}{z-x}\{: \psi(x) \partial \overrightarrow{\psi(x) \psi}(w):-\partial \psi(x) \overrightarrow{\psi(x)} \psi(w)\}= \\
& =\frac{1}{2} \frac{\psi(w)}{(z-w)^{2}}+\frac{\partial \psi(w)}{z-w},
\end{aligned}
$$

где подразумевается, что $\psi(w)$ - примарное поле с конформной размерностью $1 / 2$. Данная формула верна, поскольку разность в подынтегральном выражении можно записать как

$$
\begin{aligned}
: \psi(x) \partial \overrightarrow{\psi(x) \psi}(w):-\partial \psi(x) \overrightarrow{\psi(x) \psi}(w) & =-\frac{\psi(x)}{(x-w)^{2}}-\frac{\partial \psi(x)}{x-w}= \\
& =-\frac{\psi(w)}{(x-w)^{2}}-\frac{2 \partial \psi(w)}{x-w}+\cdots
\end{aligned}
$$

3.3. Пример 3: система духов. В этом пункте мы применяем обощенные теоремы Вика (19), (20) к так называемой системе духов $(b, c)$ [2], [7]-[9].

Пусть $b, c$ - пара фермионых полей, удовлетворяющих уравнениям

$$
\overrightarrow{b(z) b}(w)=0, \quad \overrightarrow{c(z) c}(w)=0, \quad \overrightarrow{b(z) c}(w)=\frac{1}{z-w} .
$$

Введем обозначения

$$
J(z)=: b(z) c(z):, \quad A(z)=: b^{\prime}(z) c(z):, \quad B(z)=-: b(z) c^{\prime}(z): .
$$

С учетом соотношения (19) имеем

$$
\overrightarrow{b(z) J}(w)=-\frac{b(w)}{z-w}, \quad \overrightarrow{c(z) J}(w)=\frac{c(w)}{z-w},
$$

кроме того,

$$
\begin{array}{ll}
\overrightarrow{b(z) A}(w)=-\frac{b^{\prime}(w)}{z-w}, & \overrightarrow{b(z) B}(w)=\frac{b(w)}{(z-w)^{2}}, \\
\overrightarrow{c(z) A}(w)=\frac{c(w)}{(z-w)^{2}}, & \overrightarrow{c(z) B}(w)=-\frac{c^{\prime}(w)}{z-w} .
\end{array}
$$

Отсюда с помощью соотношений (20) и (34) получаем

$$
\begin{aligned}
\overrightarrow{J(z) J}(w) & =\frac{1}{2 \pi \sqrt{-1}} \oint_{C_{w}} \frac{d x}{z-x}\{: b(x) c(x) J(w):-c(x) b(x) J(w)\}= \\
& =\frac{1}{2 \pi \sqrt{-1}} \oint_{C_{w}} \frac{d x}{z-x}\left\{\frac{: b(x) c(w):}{x-w}+\frac{c(x) b(w)}{x-w}\right\}= \\
& =\frac{1}{2 \pi \sqrt{-1}} \oint_{C_{w}} \frac{d x}{z-x}\left\{\frac{1}{(x-w)^{2}}+\frac{: b(x) c(w):+: c(x) b(w):}{x-w}\right\}= \\
& =\frac{1}{2 \pi \sqrt{-1}} \oint_{C_{w}} \frac{d x}{z-x}\left\{\frac{1}{(x-w)^{2}}+\frac{: b(w) c(w):+: c(w) b(w):}{x-w}+\cdots\right\}= \\
& =\frac{1}{(z-w)^{2}} .
\end{aligned}
$$

Таким образом, $J(z)$ - бозонный ток. 
Также, используя (20), имеем

$$
\begin{aligned}
\overrightarrow{A(z) b(w)} & =\frac{1}{2 \pi \sqrt{-1}} \oint_{C_{w}} \frac{d x}{z-x}\left\{: b^{\prime}(x) \overrightarrow{c(x) b}(w):-c(x) b^{\prime}(x) b(w)\right\}= \\
& =\frac{1}{2 \pi \sqrt{-1}} \oint_{C_{w}} \frac{d x}{z-x} \frac{b^{\prime}(x)}{x-w}=\frac{b^{\prime}(w)}{z-w}, \\
\overparen{B(z) b(w)} & =\frac{-1}{2 \pi \sqrt{-1}} \oint_{C_{w}} \frac{d x}{z-x}\left\{: b(x) c^{\prime}(x) b(w):-c^{\prime}(x) b(x) b(w)\right\}= \\
& =\frac{1}{2 \pi \sqrt{-1}} \oint_{C_{w}} \frac{d x}{z-x} \frac{b(x)}{(x-w)^{2}}=\frac{b(w)}{(z-w)^{2}}+\frac{b^{\prime}(w)}{z-w}, \\
A_{(z) c(w)} & =\frac{1}{2 \pi \sqrt{-1}} \oint_{C_{w}} \frac{d x}{z-x}\left\{: b^{\prime}(x) \overline{c(x) c}(w):-c(x) b^{\prime}(x) c(w)\right\}= \\
& =\frac{1}{2 \pi \sqrt{-1}} \oint_{C_{w}} \frac{d x}{z-x} \frac{c(x)}{(x-w)^{2}}=\frac{c(w)}{(z-w)^{2}}+\frac{c^{\prime}(w)}{z-w}, \\
B(z) c(w) & =\frac{-1}{2 \pi \sqrt{-1}} \oint_{C_{w}} \frac{d x}{z-x}\left\{: b(x) c^{\prime}(x) c(w):-c^{\prime}(x) b(x) c(w)\right\}= \\
& =\frac{1}{2 \pi \sqrt{-1}} \oint_{C_{w}} \frac{d x}{z-x} \frac{c^{\prime}(x)}{x-w}=\frac{c^{\prime}(w)}{z-w} .
\end{aligned}
$$

Тем же способом получаем, что

$$
\begin{aligned}
& \overparen{A(z) J}(w)=\frac{1}{2 \pi \sqrt{-1}} \oint_{C_{w}} \frac{d x}{z-x}\left\{: b^{\prime}(x) \overrightarrow{c(x) J}(w):-c(x) \overleftrightarrow{b^{\prime}(x) J}(w)\right\}= \\
& =\frac{1}{2 \pi \sqrt{-1}} \oint_{C_{w}} \frac{d x}{z-x}\left\{\frac{: b^{\prime}(x) c(w):}{x-w}-\frac{c(x) b(w)}{(x-w)^{2}}\right\}= \\
& =\frac{1}{2 \pi \sqrt{-1}} \oint_{C_{w}} \frac{d x}{z-x}\left\{\frac{: b^{\prime}(x) c(w):}{x-w}-\frac{1}{(x-w)^{3}}+\frac{: b(w) c(x):}{(x-w)^{2}}\right\}= \\
& =\frac{1}{2 \pi \sqrt{-1}} \oint_{C_{w}} \frac{d x}{z-x}\left\{-\frac{1}{(x-w)^{3}}+\frac{: b(w) c(w):}{(x-w)^{2}}+\right. \\
& \left.+\frac{: b^{\prime}(w) c(w):+: b(w) c^{\prime}(w):}{x-w}+\cdots\right\}= \\
& =\frac{-1}{(z-w)^{3}}+\frac{J(w)}{(z-w)^{2}}+\frac{J^{\prime}(w)}{z-w}, \\
& \overparen{B(z) J}(w)=\frac{1}{2 \pi \sqrt{-1}} \oint_{C_{w}} \frac{d x}{z-x}\left\{-: b(x) c^{\prime}(x) J(w):+c^{\prime}(x) \overrightarrow{b(x)} J(w)\right\}= \\
& =\frac{1}{2 \pi \sqrt{-1}} \oint_{C_{w}} \frac{d x}{z-x}\left\{\frac{: b(x) c(w):}{(x-w)^{2}}-\frac{c^{\prime}(x) b(w)}{x-w}\right\}= \\
& =\frac{1}{2 \pi \sqrt{-1}} \oint_{C_{w}} \frac{d x}{z-x}\left\{\frac{: b(x) c(w):}{(x-w)^{2}}+\frac{1}{(x-w)^{3}}-\frac{: c^{\prime}(x) b(w):}{x-w}\right\}= \\
& =\frac{1}{2 \pi \sqrt{-1}} \oint_{C_{w}} \frac{d x}{z-x}\left\{\frac{1}{(x-w)^{3}}+\frac{: b(w) c(w):}{(x-w)^{2}}+\right. \\
& \left.+\frac{: b^{\prime}(w) c(w):+: b(w) c^{\prime}(w):}{x-w}+\cdots\right\}= \\
& =\frac{1}{(z-w)^{3}}+\frac{J(w)}{(z-w)^{2}}+\frac{J^{\prime}(w)}{z-w} \text {. }
\end{aligned}
$$


Положим для любого $\lambda \in \mathbb{C}$

$$
T(z)=(1-\lambda) A(z)+\lambda B(z)=(1-\lambda): b^{\prime}(z) c(z):-\lambda: b(z) c^{\prime}(z): .
$$

Тогда имеем равенства

$$
\begin{aligned}
& \overparen{T(z) b}(w)=\frac{\lambda}{(z-w)^{2}} b(w)+\frac{1}{z-w} b^{\prime}(w), \\
& \overparen{T(z) c}(w)=\frac{(1-\lambda)}{(z-w)^{2}} c(w)+\frac{1}{z-w} c^{\prime}(w),
\end{aligned}
$$

а также равенство

$$
\overparen{T(z) J}(w)=\frac{2 \lambda-1}{(z-w)^{3}}+\frac{1}{(z-w)^{2}} J(w)+\frac{1}{z-w} J^{\prime}(w) .
$$

Следовательно, по отношению к введенному выше тензору энергии-импульса $T(z)$ поля $b$ и $c$ ведут себя как примарные поля с конформными размерностями $\lambda$ и $1-\lambda$ соответственно. Ток $J$ имеет конформную размерность 1 , но не является примарным полем, за исключением случая $\lambda=1 / 2[9]$.

Теперь, используя (20) и (35), мы получаем

$$
\begin{aligned}
& \overparen{A(z) A}(w)=\frac{1}{2 \pi \sqrt{-1}} \oint_{C_{w}} \frac{d x}{z-x}\left\{: b^{\prime}(x) \overrightarrow{c(x) A}(w):-c(x) \overleftrightarrow{b^{\prime}(x) A}(w)\right\}= \\
& =\frac{1}{2 \pi \sqrt{-1}} \oint_{C_{w}} \frac{d x}{z-x}\left\{\frac{: b^{\prime}(x) c(w):}{(x-w)^{2}}-\frac{c(x) b^{\prime}(w)}{(x-w)^{2}}\right\}= \\
& =\frac{1}{2 \pi \sqrt{-1}} \oint_{C_{w}} \frac{d x}{z-x}\left\{-\frac{1}{(x-w)^{4}}+\frac{: b^{\prime}(x) c(w):+: b^{\prime}(w) c(x):}{(x-w)^{2}}\right\}= \\
& =\frac{1}{2 \pi \sqrt{-1}} \oint_{C_{w}} \frac{d x}{z-x}\left\{-\frac{1}{(x-w)^{4}}+\frac{2: b^{\prime}(w) c(w):}{(x-w)^{2}}+\right. \\
& \left.+\frac{: b^{\prime \prime}(w) c(w):+: b^{\prime}(w) c^{\prime}(w):}{x-w}+\cdots\right\}= \\
& =-\frac{1}{(z-w)^{4}}+\frac{2 A(w)}{(z-w)^{2}}+\frac{A^{\prime}(w)}{z-w}, \\
& \overrightarrow{A(z) B}(w)=\frac{1}{2 \pi \sqrt{-1}} \oint_{C_{w}} \frac{d x}{z-x}\left\{: b^{\prime}(x) \overrightarrow{c(x) B}(w):-c(x) \overrightarrow{b^{\prime}(x) B}(w)\right\}= \\
& =\frac{1}{2 \pi \sqrt{-1}} \oint_{C_{w}} \frac{d x}{z-x}\left\{-\frac{: b^{\prime}(x) c^{\prime}(w):}{x-w}+\frac{2 c(x) b(w)}{(x-w)^{3}}\right\}= \\
& =\frac{1}{2 \pi \sqrt{-1}} \oint_{C_{w}} \frac{d x}{z-x}\left\{\frac{2}{(x-w)^{4}}-\frac{2: b(w) c(x):}{(x-w)^{3}}-\frac{: b^{\prime}(x) c^{\prime}(w):}{x-w}\right\}= \\
& =\frac{1}{2 \pi \sqrt{-1}} \oint_{C_{w}} \frac{d x}{z-x}\left\{\frac{2}{(x-w)^{4}}-\frac{2: b(w) c(w):}{(x-w)^{3}}-\frac{2: b(w) c^{\prime}(w):}{(x-w)^{2}}-\right. \\
& \left.-\frac{: b^{\prime}(w) c^{\prime}(w):+: b(w) c^{\prime \prime}(w):}{x-w}+\cdots\right\}= \\
& =\frac{2}{(z-w)^{4}}-\frac{2 J(w)}{(z-w)^{3}}+\frac{2 B(w)}{(z-w)^{2}}+\frac{B^{\prime}(w)}{z-w},
\end{aligned}
$$




$$
\begin{aligned}
& \overparen{B(z) A}(w)=\frac{1}{2 \pi \sqrt{-1}} \oint_{C_{w}} \frac{d x}{z-x}\left\{-: b(x) \overleftrightarrow{c^{\prime}(x) A}(w):+c^{\prime}(x) \overrightarrow{b(x) A}(w)\right\}= \\
& =\frac{1}{2 \pi \sqrt{-1}} \oint_{C_{w}} \frac{d x}{z-x}\left\{\frac{2: b(x) c(w):}{(x-w)^{3}}-\frac{c^{\prime}(x) b^{\prime}(w)}{x-w}\right\}= \\
& =\frac{1}{2 \pi \sqrt{-1}} \oint_{C_{w}} \frac{d x}{z-x}\left\{\frac{2}{(x-w)^{4}}+\frac{2: b(x) c(w):}{(x-w)^{3}}+\frac{: b^{\prime}(w) c^{\prime}(x):}{x-w}\right\}= \\
& =\frac{1}{2 \pi \sqrt{-1}} \oint_{C_{w}} \frac{d x}{z-x}\left\{\frac{2}{(x-w)^{4}}+\frac{2: b(w) c(w):}{(x-w)^{3}}+\frac{2: b^{\prime}(w) c(w):}{(x-w)^{2}}+\right. \\
& \left.+\frac{: b^{\prime \prime}(w) c(w):+: b^{\prime}(w) c^{\prime}(w):}{x-w}+\cdots\right\}= \\
& =\frac{2}{(z-w)^{4}}+\frac{2 J(w)}{(z-w)^{3}}+\frac{2 A(w)}{(z-w)^{2}}+\frac{A^{\prime}(w)}{z-w}, \\
& \overrightarrow{B(z) B}(w)=\frac{1}{2 \pi \sqrt{-1}} \oint_{C_{w}} \frac{d x}{z-x}\left\{-: b(x) \overrightarrow{c^{\prime}(x) B}(w):+c^{\prime}(x) \overrightarrow{b(x) B}(w)\right\}= \\
& =\frac{1}{2 \pi \sqrt{-1}} \oint_{C_{w}} \frac{d x}{z-x}\left\{-\frac{: b(x) c^{\prime}(w):}{(x-w)^{2}}+\frac{c^{\prime}(x) b(w)}{(x-w)^{2}}\right\}= \\
& =\frac{1}{2 \pi \sqrt{-1}} \oint_{C_{w}} \frac{d x}{z-x}\left\{-\frac{1}{(x-w)^{4}}-\frac{: b(x) c^{\prime}(w):+: b(w) c^{\prime}(x):}{(x-w)^{2}}\right\}= \\
& =\frac{1}{2 \pi \sqrt{-1}} \oint_{C_{w}} \frac{d x}{z-x}\left\{-\frac{1}{(x-w)^{4}}-\frac{2: b(w) c^{\prime}(w):}{(x-w)^{2}}-\right. \\
& \left.-\frac{: b^{\prime}(w) c^{\prime}(w):+: b(w) c^{\prime \prime}(w):}{x-w}+\cdots\right\}= \\
& =-\frac{1}{(z-w)^{4}}+\frac{2 B(w)}{(z-w)^{2}}+\frac{B^{\prime}(w)}{z-w} .
\end{aligned}
$$

Следовательно, тензор энергии-импульса (43) удовлетворяет уравнению

$$
\overparen{T(z) T}(w)=\frac{c / 2}{(z-w)^{4}}+\frac{2 T(w)}{(z-w)^{2}}+\frac{\partial T(w)}{z-w}
$$

с центральным зарядом $c=-2\left(6 \lambda^{2}-6 \lambda+1\right)[9]$.

\section{4. ЗАКЛЮЧИТЕЛЬНЫЕ ЗАМЕЧАНИЯ}

В представленной работе мы описали некоторые детали нашей новой обобщенной теоремы Вика (2) для взаимодействующих полей в двумерной КТП и представили ее обобщение (20) на модели, включающие фермионные поля. Хотя результаты, полученные в примерах 1-3, можно вывести, используя только теорему Вика для свободных (невзаимодействующих) полей, мы привели эти примеры, чтобы проиллюстрировать справедливость обощенных теорем Вика; это может оказаться полезным для читателей, которые захотят активно пользоваться нашими формулами. Эти соотношения настолько просты, что, очевидно, найдут самые многочисленные применения в различных нетривиальных задачах двумерной КТП. 


\section{Список литературы}

[1] F. A. Bais, P. Bouwknegt, M. Surridge, K. Schoutens, "Extensions of the Virasoro algebra constructed from Kac-Moody algebras using higher order Casimir invariants", Nucl. Phys. B, 304:2 (1988), 348-370.

[2] P. Di Francesco, P. Mathieu, D. Sénéchal, Conformal Field Theory, Graduate Texts in Contemporary Physics, Springer, New York, 1997.

[3] T. Takagi, T. Yoshikawa, Generalized Wick theorems in conformal field theory and the Borcherds identity, arXiv: 1604.04032 .

[4] A. Matsuo, K. Nagatomo, Axioms for a Vertex Algebra and the Locality of Quantum Fields, Mathematical Society of Japan Memoirs, 4, The Mathematical Society of Japan, Tokyo, 1999, arXiv: hep-th/9706118.

[5] R. E. Borcherds, "Vertex algebras, Kac-Moody algebras and the Monster", Proc. Nat. Acad. Sci. USA, 83:10 (1986), 3068-3071.

[6] В. Г. Кац, Вертексные алгебры для начинающих, МЦНМО, М., 2005.

[7] К. Беккер, М. Беккер, Дж. Шварц, Теория струн и М-теория. Современное введение, РХД, Ижевск, 2015.

[8] R. Blumenhagen, E. Plauschinn, Introduction to Conformal Field Theory. With Applications to String Theory, Lecture Notes in Physics, 779, Springer, Berlin, Heidelberg, 2009.

[9] Y. Yamada, Conformal Field Theory, Baifukan, Tokyo, 2006 (in Japanese). 